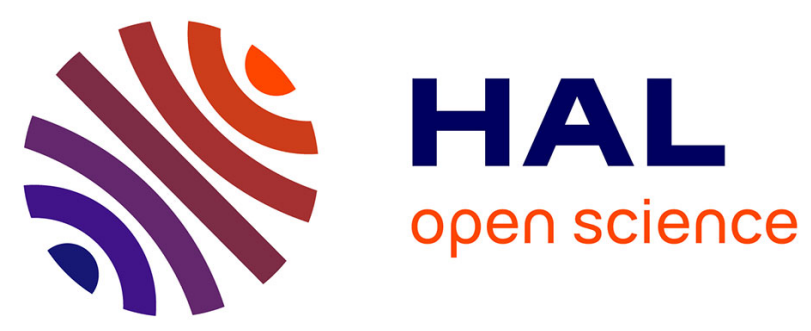

\title{
A full description of polytopes related to the index of the lowest nonzero row of an assignment matrix
}

\author{
Walid Ben-Ameur, Antoine Glorieux, José Neto
}

\section{To cite this version:}

Walid Ben-Ameur, Antoine Glorieux, José Neto. A full description of polytopes related to the index of the lowest nonzero row of an assignment matrix. ISCO 2016: 4th International Symposium on Combinatorial Optimization, May 2016, Vietri Sul Mare, Italy. pp.13-25, 10.1007/978-3-319-45587-

$7 \_2$. hal-01497880

\author{
HAL Id: hal-01497880 \\ https://hal.science/hal-01497880
}

Submitted on 29 Mar 2017

HAL is a multi-disciplinary open access archive for the deposit and dissemination of scientific research documents, whether they are published or not. The documents may come from teaching and research institutions in France or abroad, or from public or private research centers.
L'archive ouverte pluridisciplinaire HAL, est destinée au dépôt et à la diffusion de documents scientifiques de niveau recherche, publiés ou non, émanant des établissements d'enseignement et de recherche français ou étrangers, des laboratoires publics ou privés. 


\title{
A full description of polytopes related to the index of the lowest nonzero row of an assignment matrix
}

\author{
Walid Ben-Ameur, Antoine Glorieux, and José Neto \\ Samovar UMR5157, Télécom SudParis, CNRS, Universit Paris-Saclay \\ 9 Rue Charles Fourier, 91011 Evry Cedex, France \\ walid.benameur@telecom-sudparis.eu, antoine.glorieux@telecom-sudparis.eu, \\ jose.neto@telecom-sudparis.eu
}

\begin{abstract}
Consider a $\{0,1\}$ assignment matrix where each column contains exactly one coefficient equal to 1 and let $h$ be the index of the lowest row that is not identically equal to the zero row. We give a full description of the convex hull of all feasible assignments appended with the extra parameter $h$. This polytope and some of its variants naturally appear in the context of several combinatorial optimization problems including frequency assignment, job scheduling, graph orientation, maximum clique, etc. We also show that the underlying separation problems are solvable in polynomial time and thus optimization over those polytopes can be done in polynomial time.
\end{abstract}

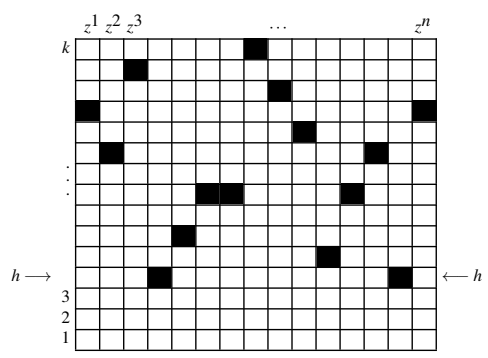

Fig. 1: On this grid representing a $k$-by$n$ matrix, each column is a $z^{i}$ variable and the height of its black cell is the value assigned to it. $h$ thus corresponds to the first non-empty row of the grid starting from the bottom (in this case, $h=4)$.

\section{Introduction and motivations}

Let us consider combinatorial optimization problems involving $n \in \mathbb{N} \backslash\{0\}$ variables $z^{i}$ each of which can be assigned an integer number in $\llbracket 1, k \rrbracket$ and let $h \equiv \min _{i=1}^{n} z^{i}$. A natural polytope related to these problems is given by

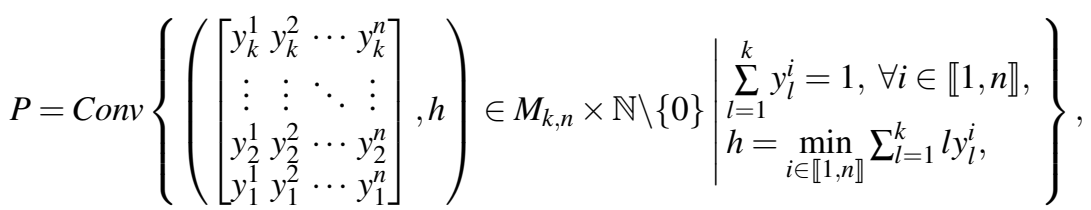


where $M_{k, n}$ is the set of all $k$-by- $n$ matrices with coefficients in $\{0,1\}$ and the variables $y_{l}^{i}$ are interpreted as follows: $y_{l}^{i}=1$ if and only if $z^{i}=l$.

The matrix $\left(y_{j}^{i}\right)$ can be seen as a $\{0,1\}$ assignment matrix where each column contains exactly one coefficient equal to 1 while $h$ denotes the index of the lowest row that is not identically equal to the zero row (cf Fig. 1).

Another variant of $P$ is obtained by considering the index of the highest row that is not identically equal to the zero row. In this case we get the polytope

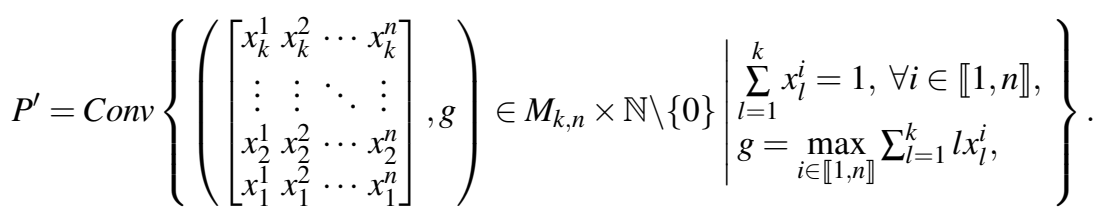

Polytopes $P$ and $P^{\prime}$ naturally appear in the context of several combinatorial optimization. Let us for example consider the minimum-span frequency-assignment problem which is a variant of the NP-hard frequency-assignment problem [8]. Given a simple graph $G=(V, E)$ that is generally called the interference graph, the frequency assignment problem consists in assigning a frequency $f$ from a set of available frequencies $F$ to each vertex $v \in V$ in such a way that each pair of antennas $u v \in E$ that may interfere with one another are assigned different frequencies. Frequencies can be seen as ordered integer numbers. To reduce interferences, one might impose stronger constraints: a minimum separation between the frequencies assigned to $u$ and $v$ is required. If frequency $i$ is assigned to $u$ and $j$ is assigned to $v$, then $|i-j| \geq s_{u v}$ where $s_{u v}$ is a given number. The minimum-span frequency-assignment problem (or MS-FAP) consists in assigning frequencies to nodes taking into account the separation requirements and minimizing the difference between the largest assigned number (frequency) and the smallest assigned number (see, e.g., [7]).

If we consider that $V=\left\{v_{1}, \cdots, v_{n}\right\}, F=\llbracket 1, k \rrbracket$ where $k$ is an upper bound of the minimum-span, then we obtain the following formulation for MS-FAP

$$
\left\{\begin{array}{l}
\min g \\
\text { s.t. } x_{l}^{i}+x_{l^{\prime}}^{j} \leq 1, \forall\left(i, j, l, l^{\prime}\right) \in \llbracket 1, n \rrbracket^{2} \times \llbracket 1, k \rrbracket^{2} \text { such that } v_{i} v_{j} \in E,\left|l-l^{\prime}\right|<s_{v_{i} v_{j}} \\
\quad(x, g) \in P^{\prime}, x \in M_{k, n} .
\end{array}\right.
$$

where the interpretation of the $x$ variable is the following: $x_{l}^{i}=1$ if and only if the frequency $l$ is assigned to the antenna $v_{i}$.

Another example is the minimum makespan scheduling, which is a central problem in the scheduling area (see [10]). Given a set $J$ of jobs, a set $M$ of machines that can all process at most one job at a time, and the time $t^{i, j} \in \mathbb{N}$ taken to process job $j \in$ $J$ on machine $i \in M$, the goal of the minimum makespan scheduling problem is to assign a machine $p \in M$ for each job $j \in J$ so as to minimize the makespan, i.e. the maximum processing time of any machine. Several approximation schemes have been developed to deal with this NP-hard problem [3], e.g. [4] and [5]. Since the processing times are integers, the timeline is discretized in identical units of time, e.g. days. We 
consider here the variant where all the machines in $M$ are identical (or IM-MMS) and preemptions are not allowed. In other words, for any job $j \in J, t^{i, j}=t^{j}, \forall i \in M$. In this case, assigning a machine to each job is equivalent to assigning a day $d^{\prime}$ to be the last day of processing this job, which also determines the first day $d$ of processing and will therefore be processed by a machine free during the period $\left[d, d^{\prime}\right]$. Now to make a formulation for IM-MMS with the set of jobs $J=\llbracket 1, n \rrbracket$ and $m \in \mathbb{N} \backslash\{0\}$ identical machines, we take $k=\sum_{i=1}^{n} t^{i}$ and the variable $x \in M_{k, n}$ whose interpretation is the following: $x_{l}^{i}=1$ if and only if the processing of the job $i$ ends on the day $l$. Then we have the following formulation for IM-MMS

$$
\left\{\begin{aligned}
\min g & \\
\text { s.t. } & \sum_{l=1}^{k} l x_{l}^{i} \geq t^{i}, \forall i \in \llbracket 1, n \rrbracket, \\
& \sum_{i=1}^{n} \sum_{l^{\prime}=l}^{\min \left(l+t^{i}-1, k\right)} x_{l^{\prime}}^{i} \leq m, \forall l \in \llbracket 1, k \rrbracket, \\
& (x, g) \in P^{\prime}, x \in M_{k, n} .
\end{aligned}\right.
$$

For a job $i \in \llbracket 1, n \rrbracket, \sum_{l=1}^{k} l x_{l}^{i} \geq t^{i}$ ensures that its processing ends after enough time has passed for $i$ to be processed, and for a day $l \in \llbracket 1, k \rrbracket, \sum_{i=1}^{n} \sum_{l^{\prime}=l}^{\min \left(l+t^{i}-1, k\right)} x_{l^{\prime}}^{i} \leq m$ ensures that no more than $m$ jobs are being processed. Some additional constraints can be added to this formulation such as a necessary precedence or release time. If we want a job $i \in \llbracket 1, n \rrbracket$ to be processed before another job $j \in \llbracket 1, n \rrbracket \backslash\{i\}$ starts processing, we add the constraint $\sum_{l=1}^{k} l x_{l}^{i} \leq \sum_{l=1}^{k} l x_{l}^{j}-t^{j}$. If we want a job $i \in \llbracket 1, n \rrbracket$ to be processed before (resp. on, after) a day $d \in \llbracket 1, k \rrbracket$, we add the constraint $\sum_{l=1}^{d-1} x_{l}^{i}=1$ (resp. $x_{d}^{i}=1$, $\left.\sum_{l=d+1}^{k} x_{l}^{i}=1\right)$. The objective function can also be any linear function depending on the $x$ and $g$ variables.

Another example is given by the problem of the most imbalanced orientation of a graph (or MAXIM) that consists in orienting the edges of a graph such that the minimum over all the vertices of the absolute difference between the outdegree and the indegree of a vertex is maximized (NP-complete) [1]. In other words, for a simple graph $G=$ $(V, E), \operatorname{MAxIM}(G)=\max _{\Lambda \in \vec{O}(G)} \min _{v \in V}\left|d_{\Lambda}^{+}(v)-d_{\Lambda}^{-}(v)\right|$, where $\vec{O}(G)$ denotes the set of all the orientations of $G$ and $d_{\Lambda}^{+}(v)$ (resp. $d_{\Lambda}^{-}(v)$ ) denotes the outdegree (resp. indegree) of $v$ in $G$ with respect to $\Lambda$. Now if we consider the graph $G$ to be arbitrarily oriented and take its incidence matrix $B \in\{-1,0,1\}^{|V| \times|E|}$, we can describe an orientation of $G$ with the variable $x \in\{-1,1\}^{|E|}$ interpreted as follows. For each edge $u v \in E$, its orientation is kept from the original one if $x_{u v}=1$ and reversed otherwise. Then if we look at the product of $B$ with an orientation vector $x \in\{-1,1\}^{|E|}$ we obtain $B_{v} x=$ $d_{x}^{+}(v)-d_{x}^{-}(v), \forall v \in V$, where $B_{v}$ denotes the row of $B$ corresponding to node $v$. In order to make a formulation of MAXIM, we consider indicator variables $t_{l}^{v} \in\{0,1\}$ with $v \in V$ and $l \in \llbracket-k, k \rrbracket, k$ being the maximum degree of the vertices of $G$, that have the following interpretation: $t_{l}^{v}=1$ if and only if $B_{v} x=d_{x}^{+}(v)-d_{x}^{-}(v)=l$, and thus we 
obtain the formulation

$$
\left\{\begin{aligned}
\max & h \\
\text { s.t. } & h \leq \sum_{l=-k}^{k}|l| t_{l}^{v}, \forall v \in V, \\
& \sum_{l=-k}^{k} t_{l}^{v}=1, \forall v \in V, \\
& \sum_{l=-k}^{k} l t_{l}^{v}=B_{v} x, \forall v \in V, \\
& x \in[-1 ; 1]^{|E|}, t \in M_{n, 2 k+1}, h \in \mathbb{R} .
\end{aligned}\right.
$$

Introducing variables $y_{l}^{v}=t_{-l}^{v}+t_{l}^{v}, \forall(v, l) \in V \times \llbracket 1, k \rrbracket$, it becomes

$$
\left\{\begin{aligned}
\max & h \\
\text { s.t. } & \sum_{l=-k}^{k} l t_{l}^{v}=B_{v} x, \forall v \in V, \\
& y_{l}^{v}=t_{-l}^{v}+t_{l}^{v}, \forall(v, l) \in V \times \llbracket 1, k \rrbracket, \\
& x \in[-1 ; 1]^{|E|},(y, h) \in P, t \in M_{n, 2 k+1} .
\end{aligned}\right.
$$

Considering the last formulation, a polyhedral analysis of the polytope $P$ may be helpful in strengthening the linear relaxation of the original formulation within the framework of a cutting-plane algorithm (see, e.g. [2] and [11]).

Polytope $P$ also appears in the context of the maximum clique problem. A discretized formulation is proposed in [9] where a variable $w_{q}^{i}$ indicates whether the vertex $i$ belongs to a clique of size $q$. These variables are of course linked to standard vertex variables ( $x_{i}=1$ if $i$ belongs to the maximum clique). The problem is then equivalent to maximizing $q$ such that $w_{q}^{i}=1$ for some $i$. This is again related to polytope $P$.

More generally, several combinatorial optimization problems where discretization techniques are used can benefit from a description of either $P$ or some of its variants.

The rest of the paper is organized as follows. First we give a complete linear description of $P$ in Section 2. Then we show that the separation problem with respect to $P$ can be solved in polynomial time in Section 3. Finally we give similar results for the polyhedron $P^{\prime}$ and others in Section 4 and conclude in Section 5.

\section{A full description of $P$}

Let us define a set of inequalities that will prove to be an hyperplane representation of $P$.

$$
\tilde{P}=\left\{\begin{array}{l}
\sum_{l=1}^{k} y_{l}^{i}=1, \forall i \in \llbracket 1, n \rrbracket, \\
\sum_{l=2}^{k} \sum_{i=1}^{n} \lambda_{l}^{i} y_{l}^{i} \geq h-1, \forall \lambda \in \Lambda, \\
\sum_{l=1}^{h_{\max }-1} \sum_{i=1}^{n}\left(l-h_{\max }\right) y_{l}^{i}+h_{\max } \leq h, \forall h_{\max } \in \llbracket 1, k \rrbracket, \\
y_{l}^{i} \geq 0, \forall(i, l) \in \llbracket 1, n \rrbracket \times \llbracket 1, k \rrbracket, h \in \mathbb{R},
\end{array}\right.
$$

where

$$
\Lambda=\left\{\begin{array}{ll}
\lambda=\left(\lambda_{l}^{i}\right)_{(i, l) \in \llbracket 1, n \rrbracket \times \llbracket 1, k \rrbracket} \in \mathbb{N}^{n k} & \begin{array}{l}
\lambda_{l+1}^{i} \geq \lambda_{l}^{i}, \forall(i, l) \in \llbracket 1, n \rrbracket \times \llbracket 1, k-1 \rrbracket \\
\sum_{i=1}^{n} \lambda_{l}^{i}=l-1, \forall l \in \llbracket 1, k \rrbracket
\end{array}
\end{array}\right\} .
$$


Any element $\lambda$ of $\Lambda$ can be constructed as follows: we start with $\lambda_{1}^{i}=0, \forall i \in \llbracket 1, n \rrbracket$, choose an index $i_{2} \in \llbracket 1, n \rrbracket$ and set $\lambda_{2}^{i_{2}}=1$ and $\lambda_{2}^{i}=0, \forall i \in \llbracket 1, n \rrbracket \backslash\left\{i_{2}\right\}$. And we proceed like this for $l=2, \cdots, k$, we choose an index $i_{l} \in \llbracket 1, n \rrbracket$ and set $\lambda_{l}^{i_{l}}=\lambda_{l-1}^{i_{l}}+1$ and $\lambda_{l}^{i}=\lambda_{l-1}^{i}, \forall i \in \llbracket 1, n \rrbracket \backslash\left\{i_{l}\right\}$.

\section{Lemma 1}

$$
P \subseteq \tilde{P}
$$

Proof. Since $P$ is the convex hull of integer points, it suffices to show that each of those points satisfies all the inequalities in $\tilde{P}$. Let $(y, h) \in P$ be one of those points, that is to say $(y, h) \in M_{k, n} \times \mathbb{N} \backslash\{0\}, \sum_{l=1}^{k} y_{l}^{i}=1, \forall i \in \llbracket 1, n \rrbracket$ and $h=\min _{i \in \llbracket 1, n \rrbracket} \sum_{l=1}^{k} l y_{l}^{i}$. We firstly show that $\sum_{l=2}^{k} \sum_{i=1}^{n} \lambda_{l}^{i} y_{l}^{i} \geq h-1, \forall \lambda \in \Lambda$. For all $i \in \llbracket 1, n \rrbracket$, there exists $l_{i} \in \llbracket h, k \rrbracket$ such that $y_{l_{i}}^{i}=1$. Now since for a given $i \in \llbracket 1, n \rrbracket, \lambda_{l}^{i}$ increases with $l$, we have

$$
\sum_{l=2}^{k} \sum_{i=1}^{n} \lambda_{l}^{i} y_{l}^{i}=\sum_{i=1}^{n} \lambda_{l_{i}^{i}} \geq \sum_{i=1}^{n} \lambda_{h}^{i}=h-1
$$

Now we take $h_{\max } \in \llbracket 1, k \rrbracket$, and we show that $h_{\max }-\sum_{l=1}^{h_{\max }-1}\left(h_{\max }-l\right) \sum_{i=1}^{n} y_{l}^{i} \leq h$. We have

$$
\begin{aligned}
h_{\max }-\sum_{l=1}^{h_{\max }-1}\left(h_{\max }-l\right) \sum_{i=1}^{n} y_{l}^{i} & =h_{\max }+\sum_{l=1}^{k} \min \left(l-h_{\max }, 0\right) \sum_{i=1}^{n} y_{l}^{i} \\
& =h_{\max }+\sum_{l=1}^{k} \min \left(l-1, h_{\max }-1\right) \sum_{i=1}^{n} y_{l}^{i}-n\left(h_{\max }-1\right) \\
& =\sum_{i=1}^{n} \sum_{l=2}^{k} \min \left(l-1, h_{\max }-1\right) y_{l}^{i}+1-(n-1)\left(h_{\max }-1\right)
\end{aligned}
$$

There exists $i^{*} \in \llbracket 1, n \rrbracket$ such that $y_{h}^{i^{*}}=1$, then

$$
\begin{array}{rlrl}
\sum_{i=1}^{n} \sum_{l=2}^{k} \min \left(l-1, h_{\max }-1\right) y_{l}^{i} & =\sum_{\substack{i=1 \\
i \neq i^{*}}}^{n} \sum_{l=2}^{k} \min \left(l-1, h_{\max }-1\right) y_{l}^{i} & & +\sum_{l=2}^{k} \min \left(l-1, h_{\max }-1\right) y_{l}^{i^{*}} \\
& \leq \sum_{\substack{i=1 \\
i \neq i^{*}}}^{n} \sum_{l=2}^{k}\left(h_{\max }-1\right) y_{l}^{i} & & +\sum_{l=2}^{k}(l-1) y_{l}^{i^{*}} \\
& \leq \sum_{\substack{i=1 \\
i \neq i^{*}}}^{n}\left(h_{\max }-1\right) & & +h-1 \\
& =(n-1)\left(h_{\max }-1\right) & +h-1 .
\end{array}
$$

Now to prove that $P$ coincides with $\tilde{P}$, we show that all facet-defining inequalities for $P$ are among those defining $\tilde{P}$. Two inequalities are said to be equivalent if one can be obtained from the other by multiplying it by a non-zero scalar and adding a combination of equations of the type $\sum_{l=1}^{k} y_{l}^{i}=1$. 


\section{Lemma 2 Let}

$$
\sum_{l=1}^{k} \sum_{i=1}^{n} \beta_{l}^{i} y_{l}^{i}+\gamma \geq 0
$$

be a facet-defining inequality of $P$, with $\beta_{l}^{i} \in \mathbb{R}, \forall(i, l) \in \llbracket 1, n \rrbracket \times \llbracket 1, k \rrbracket, \gamma \in \mathbb{R}$. Then there exists $(i, l) \in \llbracket 1, n \rrbracket \times \llbracket 1, k \rrbracket$ such that (1) is equivalent to $y_{l}^{i} \geq 0$.

For an extreme point $(y, h)$ of $P$ and $\left(\tilde{i}, \tilde{l}, \tilde{l}^{\prime}\right) \in \llbracket 1, n \rrbracket \times \llbracket 1, k \rrbracket^{2}$, such that $y_{\tilde{l}}^{\tilde{i}}=1$ and $\tilde{l} \neq$ $\tilde{l}^{\prime}$, we denote by $\left(y_{\tilde{l} \tilde{i}} \tilde{l}^{\prime}, h_{\tilde{l} \stackrel{i}{\longrightarrow} \tilde{l}^{\prime}}\right)$ the extreme point $\left(y^{\prime}, h^{\prime}\right)$ of $P$ such that $y_{l}^{\prime i}=y_{l}^{i}, \forall(i, l) \in$ $\llbracket 1, n \rrbracket \times \llbracket 1, k \rrbracket \backslash\left\{(\tilde{i}, \tilde{l}),\left(\tilde{i}, \tilde{l^{\prime}}\right)\right\}, y_{\tilde{l}}^{\prime \tilde{i}}=0, y_{\tilde{l}^{\prime}}^{\prime \tilde{i}}=1$ and $h^{\prime}=\min _{i \in \llbracket 1, n \rrbracket} \sum_{l=1}^{k} l y_{l}^{\prime i}$. For $\tilde{l} \in \llbracket 1, k \rrbracket$, we denote by $\left(y_{\rightarrow \tilde{l}}, \tilde{l}\right)$ the point of $P$ such that $y_{\tilde{l}}^{i}=1, \forall i \in \llbracket 1, n \rrbracket$ and $y_{l}^{i}=0, \forall(i, l) \in$ $\llbracket 1, n \rrbracket \times(\llbracket 1, k \rrbracket \backslash\{\tilde{l}\})$.

Proof. First, since for each $i \in \llbracket 1, n \rrbracket$, we have $\sum_{l=1}^{k} y_{l}^{i}=1$, we can replace $y_{1}^{i}$ by $1-$ $\sum_{l=2}^{k} y_{l}^{i}$ and get new coefficients $\tilde{\beta}_{1}^{i}=0$ and $\tilde{\beta}_{l}^{i}=\beta_{l}^{i}-\beta_{1}^{i}, \forall l \in \llbracket 2, k \rrbracket$ and a new $\tilde{\gamma}=\gamma+$ $\sum_{i=1}^{n} \beta_{1}^{i}$. Hence, without loss of generality, we can assume that $\beta_{1}^{i}=0$ for all $i \in \llbracket 1, n \rrbracket$. Suppose that the facet defined by (1) is not equivalent to a facet defined by an inequality of the type $y_{l}^{i} \geq 0$. If we take $(\tilde{i}, \tilde{l}) \in \llbracket 1, n \rrbracket \times \llbracket 1, k-1 \rrbracket$, we know that there exists an extreme point $(y, h)$ of $P$ saturating (1) and such that $y_{\tilde{l}}^{\tilde{i}}=1$, otherwise all the extreme points saturating (1) would saturate $y_{\tilde{l}}^{\tilde{i}} \geq 0$ thus contradicting the fact that (1) is facetdefining and not equivalent to $y_{l}^{i} \geq 0$ for some $(i, l) \in \llbracket 1, n \rrbracket \times \llbracket 1, k \rrbracket$. Since $\left(y^{\prime}, h^{\prime}\right)=$ $\left(y_{\tilde{l} \stackrel{\tilde{i}}{\rightarrow} \tilde{l}+1}, h_{\tilde{l} \tilde{i}_{i} \tilde{l}+1}\right) \in P$, we have $\sum_{l=1}^{k} \sum_{i=1}^{n} \beta_{l}^{i} y_{l}^{i}+\gamma=0$ and $\sum_{l=1}^{k} \sum_{i=1}^{n} \beta_{l}^{i} y_{l}^{\prime i}+\gamma \geq 0$ which yields $\beta_{\tilde{l}+1}^{\tilde{i}} \geq \beta_{\tilde{l}}^{\tilde{i}}$. Similarly, taking an extreme point $(y, h)$ of $P$ saturating (1) and

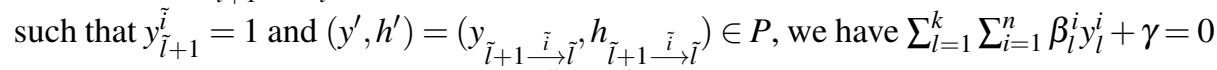
and $\sum_{l=1}^{k} \sum_{i=1}^{n} \beta_{l}^{i} y_{l}^{i i}+\gamma \geq 0$, yielding $\beta_{\tilde{l}}^{\tilde{i}} \geq \beta_{\tilde{l}+1}^{\tilde{i}}$. Hence, for all $(i, l) \in \llbracket 1, n \rrbracket \times \llbracket 1, k-1 \rrbracket$, we have $\beta_{l}^{i}=\beta_{l+1}^{i}$, in other words, $\beta_{l}^{i}=0, \forall(i, l) \in \llbracket 1, n \rrbracket \times \llbracket 1, k \rrbracket$, and (1) is not facetdefining.

Lemma 3 Let

$$
\sum_{l=1}^{k} \sum_{i=1}^{n} \beta_{l}^{i} y_{l}^{i}+\gamma \geq h
$$

be a facet-defining inequality of $P$, with $\beta_{l}^{i} \in \mathbb{R}, \forall(i, l) \in \llbracket 1, n \rrbracket \times \llbracket 1, k \rrbracket, \gamma \in \mathbb{R}$. Then there exists $\lambda \in \Lambda$ such that (2) is equivalent to $\sum_{l=2}^{k} \sum_{i=1}^{n} \lambda_{l}^{i} y_{l}^{i} \geq h-1$.

Proof. Again, without loss of generality, we assume that $\beta_{1}^{i}=0$ for all $i \in \llbracket 1, n \rrbracket$. For an $(\tilde{i}, \tilde{l}) \in \llbracket 1, n \rrbracket \times \llbracket 1, k-1 \rrbracket$, there exists an extreme point $(y, h)$ of $P$ saturating $(2)$ and such that $y_{\tilde{l}}^{\tilde{i}}=1$. Since $\left(y^{\prime}, h^{\prime}\right)=\left(y_{\tilde{l} \stackrel{\tilde{i}}{\longrightarrow} \tilde{l}+1}, h_{\tilde{l} \stackrel{\tilde{i}}{\longrightarrow} \tilde{l}+1}\right) \in P$, we have $\sum_{l=1}^{k} \sum_{i=1}^{n} \beta_{l}^{i} y_{l}^{i}+\gamma=h$ and $\sum_{l=1}^{k} \sum_{i=1}^{n} \beta_{l}^{i} y_{l}^{\prime i}+\gamma \geq h^{\prime} \geq h$ which yields $\beta_{\tilde{l}+1}^{\tilde{i}} \geq \beta_{\tilde{l}}^{\tilde{i}}$. Hence for all $i \in \llbracket 1, n \rrbracket,\left(\beta_{l}^{i}\right)_{l}$ is increasing with $l$ and therefore non-negative since $\beta_{1}^{i}=0, \forall i \in \llbracket 1, n \rrbracket$.

If we consider the point $(y, h)=\left(y_{\rightarrow 1}, 1\right)$, we obtain that $\gamma \geq 1$. If we now consider an 
extreme point $(y, h)$ of $P$ saturating (2) and such that $y_{1}^{\tilde{i}}=1$ for an $\tilde{i} \in \llbracket 1, n \rrbracket$, we get $\sum_{l=1}^{k} \sum_{i=1}^{n} \beta_{l}^{i} y_{l}^{\prime i}+\gamma=h=1$. But since both the $\beta_{l}^{i}$ and the $y_{l}^{i}$ are non-negative, then so is $\sum_{l=1}^{k} \sum_{i=1}^{n} \beta_{l}^{i} y_{l}^{\prime i}$. Hence $\gamma \leq 1$, yielding $\gamma=1$.

Considering $\left(y_{\rightarrow l}, l\right) \in P$ for $l \in \llbracket 1, k \rrbracket$, we obtain $\sum_{i=1}^{n} \beta_{l}^{i} \geq l-1$. Let us show by induction on $l$ that $\sum_{i=1}^{n} \beta_{l}^{i}=l-1, \forall l \in \llbracket 1, k \rrbracket$. Our induction is already initialized by $\sum_{i=1}^{n} \beta_{1}^{i}=0$. We suppose that for a $\tilde{l} \in \llbracket 1, k-1 \rrbracket$, we have $\sum_{i=1}^{n} \beta_{\tilde{l}}^{i}=\tilde{l}-1$ and show that $\sum_{i=1}^{n} \beta_{\tilde{l}+1}^{i}=\tilde{l}$. Suppose that all the extreme points $(y, h)$ of $P$ saturating (2) and such that $y_{\tilde{l}+1}^{i}=1$ for some $i \in \llbracket 1, n \rrbracket$ verify $h \leq \tilde{l}$. Then for each $\tilde{i} \in \llbracket 1, n \rrbracket$, take one of those extreme saturating points such that $y_{\tilde{l}+1}^{\tilde{i}}=1$ and let $\left(y^{\prime}, h^{\prime}\right)=\left(y_{\tilde{l}+1 \stackrel{\tilde{i}}{\longrightarrow} \tilde{l}}, h_{\tilde{l}+1 \stackrel{\tilde{i}}{\longrightarrow} \tilde{l}}\right) \in P$. Since $\beta_{\tilde{l}+1}^{\tilde{i}} \geq \beta_{\tilde{l}}^{\tilde{i}}$, we have $h^{\prime}-1 \leq \sum_{l=1}^{k} \sum_{i=1}^{n} \beta_{l}^{i} y_{l}^{\prime i} \leq \sum_{l=1}^{k} \sum_{i=1}^{n} \beta_{l}^{i} y_{l}^{i}=h-1$, and since $h \leq \tilde{l}$, we have $h=h^{\prime}$ and therefore, $\sum_{l=1}^{k} \sum_{i=1}^{n} \beta_{l}^{i} y_{l}^{\prime i}=\sum_{l=1}^{k} \sum_{i=1}^{n} \beta_{l}^{i} y_{l}^{i}=h-1$, yielding $\beta_{\tilde{l}+1}^{\tilde{i}}=\beta_{\tilde{l}}^{\tilde{i}}$. Thus $\sum_{i=1}^{n} \beta_{\tilde{l}+1}^{i}=\sum_{i=1}^{n} \beta_{\tilde{l}}^{i}=\tilde{l}-1$ which contradicts $\sum_{i=1}^{n} \beta_{\tilde{l}+1}^{i} \geq \tilde{l}$. So there exists $\tilde{i} \in \llbracket 1, n \rrbracket$ and $(y, h)$ an extreme point of $P$ saturating (2) such that $y_{\tilde{l}+1}^{\tilde{i}}=1$ and $h=\tilde{l}+1$. We have $\tilde{l} \leq \sum_{i=1}^{n} \beta_{\tilde{l}+1}^{i} \leq \sum_{l=1}^{k} \sum_{i=1}^{n} \beta_{l}^{i} y_{l}^{i}=\tilde{l}$, hence $\sum_{i=1}^{n} \beta_{\tilde{l}+1}^{i}=\tilde{l}$, which concludes our induction.

Now let us show by induction on $l$ that for all $(i, l) \in \llbracket 1, n \rrbracket \times \llbracket 1, k \rrbracket, \beta_{l}^{i}$ is an integer. This induction is trivially initialized for $\beta_{1}^{i}=0, \forall i \in \llbracket 1, n \rrbracket$. We suppose that for a $\tilde{l} \in$ $\llbracket 1, k-1 \rrbracket$ we have that the $\beta_{\tilde{l}}^{i}$ for $i \in \llbracket 1, n \rrbracket$ are integers and we show that the same holds for the $\beta_{\tilde{l}+1}^{i}$ for $i \in \llbracket 1, n \rrbracket$. We note $\alpha^{i}=\beta_{\tilde{l}+1}^{i}-\beta_{\tilde{l}}^{i}, \forall i \in \llbracket 1, n \rrbracket$ and for each $i \in \llbracket 1, n \rrbracket$ we build a new set of inequality coefficients : $\beta_{l}^{(i), j}=\beta_{l}^{j}-\alpha^{j}+\delta_{i, j}, \forall(j, l) \in \llbracket 1, n \rrbracket \times \llbracket 1, k \rrbracket$, where $\delta_{i, j}$ equals 1 if $i=j$ and 0 otherwise. Let $(y, h)$ be an extreme point of $P$ and for all $j \in \llbracket 1, n \rrbracket$, let $l_{j} \in \llbracket h, k \rrbracket$ be such that $y_{l_{j}}^{j}=1$. Then, since $\sum_{i=1}^{n} \alpha^{i}=1$, we have for $i \in \llbracket 1, n \rrbracket$

$\sum_{l=1}^{k} \sum_{j=1}^{n} \beta_{l}^{(i), j} y_{l}^{j}=\sum_{l=1}^{k} \sum_{j=1}^{n}\left(\beta_{l}^{j}-\alpha^{j}+\delta_{i, j}\right) y_{l}^{j}=\sum_{j=1}^{n}\left(\beta_{l_{j}}^{j}-\alpha^{j}+\delta_{i, j}\right)=\sum_{j=1}^{n} \beta_{l_{j}}^{j} \geq \sum_{i=1}^{n} \beta_{h}^{i}=h-1$,

which means that for all $i \in \llbracket 1, n \rrbracket, \sum_{l=1}^{k} \sum_{j=1}^{n} \beta_{l}^{(i), j} y_{l}^{j}+1 \geq h$ is valid for $P$. Now since for $(j, l) \in \llbracket 1, n \rrbracket \times \llbracket 1, k \rrbracket$,

$$
\left(\sum_{i=1}^{n} \alpha^{i} \beta^{(i)}\right)_{l}^{j}=\sum_{i=1}^{n} \alpha^{i}\left(\beta_{l}^{j}-\alpha^{j}+\delta_{i, j}\right)=\left(\sum_{i=1}^{n} \alpha^{i}\right) \beta_{l}^{j}-\left(\sum_{i=1}^{n} \alpha^{i}\right) \alpha^{j}+\sum_{i=1}^{n} \alpha^{i} \delta_{i, j}=\beta_{l}^{j}
$$

and $\alpha^{i} \geq 0, \forall i \in \llbracket 1, n \rrbracket,(2)$ is a convex combination of these inequalities. Moreover, if any of the $\beta_{l+1}^{i}, i \in \llbracket 1, n \rrbracket$ was not an integer, then the convex combination would be non-trivial, which would contradict the fact that (2) is facet-defining. Concluding our induction, we obtain that for all $(i, l) \in \llbracket 1, n \rrbracket \times \llbracket 1, k \rrbracket, \beta_{l}^{i}$ is an integer. And thus that $\beta \in \Lambda$, i.e. (2) belongs to the set of inequalities defining $\tilde{P}$. 


\section{Lemma 4 Let}

$$
\sum_{l=1}^{k} \sum_{i=1}^{n} \beta_{l}^{i} y_{l}^{i}+\gamma \leq h
$$

be a facet-defining inequality of $P$, with $\beta_{l}^{i} \in \mathbb{R}, \forall(i, l) \in \llbracket 1, n \rrbracket \times \llbracket 1, k \rrbracket, \gamma \in \mathbb{R}$. Then it is equivalent to $h_{\max }-\sum_{l=1}^{h_{\max }-1}\left(h_{\max }-l\right) \sum_{i=1}^{n} y_{l}^{i} \leq h$ for some $h_{\max } \in \llbracket 1, k \rrbracket$.

Proof. Since for each $i \in \llbracket 1, n \rrbracket$, we have $\sum_{l=1}^{k} y_{l}^{i}=1$, we can replace $\beta_{l}^{i}$ by $\tilde{\beta}_{l}^{i}=\beta_{l}^{i}-v_{i}$, for some $v_{i} \geq 0$ such that $\tilde{\beta}_{l}^{i} \leq 0, \forall(i, l) \in \llbracket 1, n \rrbracket \times \llbracket 1, k \rrbracket$, and $\gamma$ by $\tilde{\gamma}=\gamma+\sum_{i=1}^{n} v_{i}$ and thus get new coefficients $\tilde{\beta}$ which are non-positive without changing (3). So without loss of generality, we can assume that $\beta$ is non-positive and furthermore that $\gamma$ is minimal for a non-positive $\beta$.

For $(\tilde{i}, \tilde{l}) \in \llbracket 1, n \rrbracket \times \llbracket 2, k \rrbracket$, we take an extreme point $(y, h)$ of $P$ saturating (3) such that $y_{\tilde{l}}^{\tilde{i}}=1$ and $\left(y^{\prime}, h^{\prime}\right)=\left(y_{\tilde{l} \stackrel{\tilde{i}}{\rightarrow} \tilde{l}-1}, h_{\tilde{l} \underline{i} \dot{l} \tilde{l}-1}\right)$. We have $\sum_{l=1}^{k} \sum_{i=1}^{n} \beta_{l}^{i} y_{l}^{i}+\gamma=h$ and $\sum_{l=1}^{k} \sum_{i=1}^{n} \beta_{l}^{i} y_{l}^{\prime i}+$ $\gamma \leq h^{\prime} \leq h$, which yields $\beta_{\tilde{l}-1}^{\tilde{i}} \leq \beta_{\tilde{l}}^{\tilde{i}}$. In other words, $\beta_{l}^{i}$ is increasing with $l$, for all $i \in \llbracket 1, n \rrbracket$. This implies that for all $i \in \llbracket 1, n \rrbracket$, there exists $l_{i} \in \llbracket 1, k \rrbracket$ such that $\beta_{l}^{i}=$ $0, \forall l \in \llbracket l_{i}, k \rrbracket$ and $\beta_{l}^{i}<0$ for $l<l_{i}$ because suppose there exists $i \in \llbracket 1, n \rrbracket$ for which $\beta_{k}^{i}<0$, then we can replace $\beta_{l}^{i}$ by $\beta_{l}^{i}-\beta_{k}^{i}$ for all $l \in \llbracket 1, k \rrbracket$ and add $\beta_{k}^{i}$ to $\gamma$ and thus get new non-positive coefficients $\tilde{\beta}$ with a $\tilde{\gamma}<\gamma$, which contradicts the minimality of $\gamma$.

Let $(\tilde{i}, \tilde{l}) \in \llbracket 1, n \rrbracket \times \llbracket 1, k \rrbracket$ such that $\beta_{\tilde{l}}^{\tilde{i}}<0$ (i.e., $\tilde{l}<l_{\tilde{i}}$ ), we know there exists an extreme point $(y, h)$ of $P$ saturating (3) such that $y_{\tilde{l}}^{\tilde{i}}=1$. Suppose that $h<\tilde{l}$, we take $\left(y^{\prime}, h^{\prime}\right)$ an extreme point of $P$ such that $y_{l}^{\prime i}=y_{l}^{i}, \forall(i, l) \in \llbracket 1, n \rrbracket \backslash\{\tilde{i}\} \times \llbracket 1, k \rrbracket, y_{l_{\tilde{i}}^{\prime}}^{\prime \tilde{i}}=1$ and obtain $h=\sum_{l=1}^{k} \sum_{i=1}^{n} \beta_{l}^{i} y_{l}^{i}+\gamma \leq \sum_{l=1}^{k} \sum_{i=1}^{n} \beta_{l}^{i} y_{l}^{\prime i}+\gamma \leq h^{\prime}=h$, that yields $\beta_{\tilde{l}}^{\tilde{i}}=0$, which is a contradiction. Hence $h=\tilde{l}$, so if we take the extreme point $\left(y^{\prime}, h^{\prime}\right)$ of $P$ such that $y_{\tilde{l}}^{\prime \tilde{i}}=1, y_{k}^{\prime i}=1, \forall i \in \llbracket 1, n \rrbracket \backslash\{\tilde{i}\}$ and $y_{l}^{i}=0, \forall(i, l) \in \llbracket 1, n \rrbracket \times \llbracket 1, k \rrbracket \backslash(\{(\tilde{i}, \tilde{l})\} \cup\{(i, k), i \in$ $\llbracket 1, n \rrbracket \backslash\{\tilde{i}\}\})$, we have

$$
\tilde{l}=\sum_{l=1}^{k} \sum_{i=1}^{n} \beta_{l}^{i} y_{l}^{i}+\gamma \leq \sum_{l=1}^{k} \sum_{i=1}^{n} \beta_{l}^{i} y_{l}^{\prime i}+\gamma=\beta_{\tilde{l}}^{\tilde{i}}+\gamma \leq h^{\prime}=\tilde{l} .
$$

This gives us that for all $i \in \llbracket 1, n \rrbracket, \beta_{l}^{i}=l-\gamma, \forall l \in \llbracket 1, l_{i}-1 \rrbracket$. Consider the extreme point $(y, h)$ of $P$ such that for all $i \in \llbracket 1, n \rrbracket, y_{l_{i}}^{i}=1$, we have $\gamma \leq \min _{i \in \llbracket 1, n \rrbracket} l_{i}$. We call $h_{\max }$ the maximum value of $h$ among the extreme points $(y, h)$ of $P$ saturating (3).

If we take an extreme point $(y, h)$ of $P$ realizing $h_{\max }$, i.e. saturating (3) and such that $h=h_{\max }$, we have $\sum_{l=1}^{k} \sum_{i=1}^{n} \beta_{l}^{i} y_{l}^{i}+\gamma=h_{\max }$ which, since $\sum_{l=1}^{k} \sum_{i=1}^{n} \beta_{l}^{i} y_{l}^{i}$ is nonpositive, yields $h_{\max } \leq \gamma$. Now for $\tilde{i} \in \llbracket 1, n \rrbracket$, there exists an extreme point $(y, h)$ of $P$ saturating (3) such that $y_{l_{\tilde{i}}-1}^{\tilde{i}}=1$. Suppose that $h<l_{\tilde{i}}-1$, we take $\left(y^{\prime}, h^{\prime}\right)$ an extreme point of $P$ such that $y_{l}^{\prime i}=y_{l}^{i}, \forall(i, l) \in \llbracket 1, n \rrbracket \backslash\{\tilde{i}\} \times \llbracket 1, k \rrbracket, y_{l_{\tilde{i}}}^{\tilde{i}}=1$ and obtain

$$
h=\sum_{l=1}^{k} \sum_{i=1}^{n} \beta_{l}^{i} y_{l}^{i}+\gamma \leq \sum_{l=1}^{k} \sum_{i=1}^{n} \beta_{l}^{i} y_{l}^{i}+\gamma \leq h^{\prime}=h,
$$


that yields $\beta_{l_{\tilde{i}}-1}^{\tilde{i}}=0$, which is a contradiction. So $h=l_{\tilde{i}}-1$, hence $h_{\max } \geq l_{\tilde{i}}-1$. We obtain $\max _{i \in \llbracket 1, n \rrbracket} l_{i}-1 \leq h_{\max } \leq \gamma \leq \min _{i \in \llbracket 1, n \rrbracket} l_{i} \leq \max _{i \in \llbracket 1, n \rrbracket} l_{i}$. There are two possibilities, either $\min _{i \in \llbracket 1, n \rrbracket} l_{i}=\max _{i \in \llbracket 1, n \rrbracket} l_{i}$, or $\max _{i \in \llbracket 1, n \rrbracket} l_{i}-1=\min _{i \in \llbracket 1, n \rrbracket} l_{i}$. Suppose $\max _{i \in \llbracket 1, n \rrbracket} l_{i}-1=h_{\max }=\gamma=\min _{i \in \llbracket 1, n \rrbracket} l_{i}$, then there exists $\tilde{i} \in \llbracket 1, n \rrbracket$ such that $l_{\tilde{i}}=1+h_{\max }$, which implies that $\beta_{h_{\max }}^{\tilde{i}} \neq 0$ and $\beta_{h_{\max }}^{\tilde{i}}=h_{\max }-\gamma=0$ which is a contradiction. So $\min _{i \in \llbracket 1, n \rrbracket} l_{i}=\max _{i \in \llbracket 1, n \rrbracket} l_{i}=: L$ and

$$
L-1 \leq h_{\max } \leq \gamma \leq L .
$$

Let us assume that $h_{\max }=L-1$. Then we know that $\gamma<L$, otherwise if $\gamma=L$, the extreme point of $P\left(y_{\rightarrow L}, L\right)$ would saturate (3) and thus contradict the maximality of $h_{\max }$. We consider the following inequality

$$
\sum_{l=1}^{L-1} \sum_{i=1}^{n}(l-L) y_{l}^{i}+L \leq h
$$

Let us show that it is a valid inequality for $P$, that is to say, that every extreme point $(y, h)$ of $P$ verifies it. If $h \geq L$, then $\sum_{l=1}^{L-1} \sum_{i=1}^{n}(l-L) y_{l}^{i}=0$ and we are done. If $h \leq L-1$, then there exists $\tilde{i} \in \llbracket 1, n \rrbracket$ such that $y_{h}^{\tilde{i}}=1$. Combining this with the validity of (3) implies

$\sum_{l=1}^{L-1} \sum_{i=1}^{n}(l-L) y_{l}^{i}+L=\sum_{l=1}^{L-1} \sum_{i=1}^{n}(l-\gamma) y_{l}^{i}+\gamma+\sum_{l=1}^{L-1} \sum_{i=1}^{n}(\gamma-L) y_{l}^{i}+L-\gamma \leq h+\gamma-L+L-\gamma=h$.

Moreover, if $(y, h)$ is an extreme point of $P$ saturating (3), then there exists $\tilde{i} \in \llbracket 1, n \rrbracket$ such that $y_{h}^{\tilde{i}}=1$ and $h \leq h_{\max }=L-1$ which yields $\sum_{l=1}^{L-1} \sum_{i=1}^{n}(l-\gamma) y_{l}^{i}+\gamma=h=$

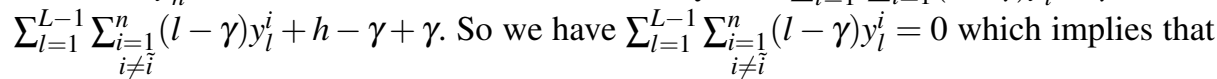
$y_{l}^{i}=0, \forall(i, l) \in(\llbracket 1, n \rrbracket \backslash\{\tilde{i}\}) \times \llbracket 1, L-1 \rrbracket$. Thus

$\sum_{l=1}^{L-1} \sum_{i=1}^{n}(l-L) y_{l}^{i}+L=\sum_{l=1}^{L-1} \sum_{i=1}^{n}(l-\gamma) y_{l}^{i}+\gamma+\sum_{l=1}^{L-1} \sum_{i=1}^{n}(\gamma-L) y_{l}^{i}+L-\gamma=h+\gamma-L+L-\gamma=h$.

Consequently, all points saturating (3) saturate (4), furthermore, $\left(y_{\rightarrow L}, L\right)$ saturates (4) and not (3). This means that the face of the polyhedron defined by (3) is strictly contained in the face defined by (4) contradicting the fact that (3) is facet-defining, hence $h_{\max }=\gamma=L$ and (3) becomes $\sum_{l=1}^{h_{\max }-1} \sum_{i=1}^{n}\left(l-h_{\max }\right) y_{l}^{i}+h_{\max } \leq h$ with $h_{\max } \in \llbracket 1, n \rrbracket$.

\section{Theorem 5}

$$
P=\tilde{P}
$$

Proof. With Lemma 1, we know that $P \subseteq \tilde{P}$. Take any facet-defining inequality of $P$ $\sum_{l=1}^{k} \sum_{i=1}^{n} \beta_{l}^{i} y_{l}^{i}+\gamma \geq \alpha h$, where $\beta_{l}^{i} \in \mathbb{R}, \forall(i, l) \in \llbracket 1, n \rrbracket \times \llbracket 1, k \rrbracket,(\gamma, \alpha) \in \mathbb{R} \times\{-1,0,1\}$. If $\alpha=0$ (resp. $\alpha=1, \alpha=-1$ ), then Lemma 2 (resp. Lemma 3, Lemma 4) gives us that this inequality is equivalent to one of those defining $\tilde{P}$. 


\section{Separation problem}

$P$ is defined by $n$ equalities, $k n$ non-negativity constraints, $k$ constraints of type $\sum_{l=1}^{h_{\max }-1} \sum_{i=1}^{n}(l-$ $\left.h_{\max }\right) y_{l}^{i}+h_{\max } \leq h$ and $n^{k-1}$ of inequalities of type $\sum_{l=2}^{k} \sum_{i=1}^{n} \lambda_{l}^{i} y_{l}^{i} \geq h-1$. The total number of inequalities is then exponential. However, the following holds.

Theorem 6 The separation problem which consists in deciding if a vector $(y, h) \in$ $\mathbb{R}^{n k+1}$ is in $P$, and if not in returning a constraint of $P$ violated by $(y, h)$ can be solved in polynomial time.

Proof. Let $(y, h) \in \mathbb{R}^{n k+1}$, first, one can verify in linear time if $(y, h) \in[0,1]^{n k} \times[1, k]$ is such that $\sum_{l=1}^{k} y_{l}^{i}=1, \forall i \in \llbracket 1, n \rrbracket$ and verifies the $k$ inequalities of type $\sum_{l=1}^{h_{\max }-1} \sum_{i=1}^{n}(l-$ $\left.h_{\max }\right) y_{l}^{i}+h_{\max } \leq h$. If not, we return a violated constraint. Otherwise, we build $\tilde{\lambda} \in \Lambda$ as follows: $\tilde{\lambda}_{1}^{i}=0, \forall i \in \llbracket 1, n \rrbracket$, and for $l=2, \cdots, k$, let $\tilde{i}_{l}=\arg \min _{i \in \llbracket 1, n \rrbracket} y_{l}^{i}+y_{l+1}^{i}+\cdots+y_{k}^{i}$ and set $\tilde{\lambda}_{l}^{\tilde{i}_{l}}=\tilde{\lambda}_{l-1}^{\tilde{i}_{l}}+1$ and $\tilde{\lambda}_{l}^{i}=\tilde{\lambda}_{l-1}^{i}, \forall i \in \llbracket 1, n \rrbracket \backslash\left\{\tilde{i}_{l}\right\}$. We will show that if $(y, h)$ satisfies the inequality of $P$ corresponding to $\tilde{\lambda}$, then it satisfies all the inequalities corresponding to an element of $\Lambda$. Suppose $\sum_{l=2}^{k} \sum_{i=1}^{n} \tilde{\lambda}_{l}^{i} y_{l}^{i} \geq h-1$ and let $\lambda \in \Lambda$ and $\left(i_{2}, \cdots, i_{k}\right) \in$ $\llbracket 1, n \rrbracket^{k-1}$ the indices corresponding to the building of $\lambda$, i.e. for $l=2, \cdots, k, \lambda_{l}^{i_{l}}=$ $\lambda_{l-1}^{i_{l}}+1$ and $\lambda_{l}^{i}=\lambda_{l-1}^{i}, \forall i \in \llbracket 1, n \rrbracket \backslash\left\{i_{l}\right\}$. By construction of $i_{2}, \cdots, i_{k}$ and $\tilde{i}_{2}, \cdots, \tilde{i}_{k}$, we have

$$
\sum_{l=2}^{k} \sum_{i=1}^{n} \lambda_{l}^{i} y_{l}^{i}=\sum_{l=2}^{k}\left(y_{l}^{i_{l}}+y_{l+1}^{i_{l}}+\cdots+y_{k}^{i_{l}}\right) \geq \sum_{l=2}^{k}\left(y_{l}^{\tilde{i}_{l}}+\tilde{y}_{l+1}^{\tilde{i}_{l}}+\cdots+y_{k}^{\tilde{i}_{l}}\right)=\sum_{l=2}^{k} \sum_{i=1}^{n} \tilde{\lambda}_{l}^{i} y_{l}^{i} \geq h-1,
$$

hence the inequality of $P$ corresponding to $\lambda$ is satisfied. So we can conclude that if $(y, h)$ satisfies the inequality of $P$ corresponding to $\tilde{\lambda}$, then it satisfies all the inequalities of $P$ corresponding to an element of $\Lambda$. And since the construction of $\tilde{\lambda}$ is done in polynomial time, the separation problem is indeed polynomial.

The previous result is very useful in the context of cutting plane algorithms where only violated inequalities are added and not all valid inequalities.

\section{Variants}

Linear programming formulations aiming to maximize (resp. minimize) the index of the lowest (resp. highest) nonzero row of an assignment matrix are related to polytope $Q$ (resp. $Q^{\prime}$ ) described below. Observe that $h$ (resp. $g$ ) is only required to be less (resp. more) than or equal to $\min _{i \in \llbracket 1, n \rrbracket} \sum_{l=1}^{k} l y_{l}^{i}\left(\right.$ resp. $\left.\max _{i \in \llbracket 1, n \rrbracket} \sum_{l=1}^{k} l x_{l}^{i}\right)$.

$$
Q=\operatorname{Conv}\left\{\left(\left[\begin{array}{cccc}
y_{k}^{1} & y_{k}^{2} & \cdots & y_{k}^{n} \\
\vdots & \vdots & \ddots & \vdots \\
y_{2}^{1} & y_{2}^{2} & \cdots & y_{2}^{n} \\
y_{1}^{1} & y_{1}^{2} & \cdots & y_{1}^{n}
\end{array}\right], h\right) \in M_{k, n} \times \mathbb{N} \backslash\{0\} \mid \begin{array}{l}
\sum_{l=1}^{k} y_{l}^{i}=1, \forall i \in \llbracket 1, n \rrbracket, \\
h \leq \min _{i \in \llbracket 1, n \rrbracket} \sum_{l=1}^{k} l y_{l}^{i},
\end{array}\right\}
$$




$$
Q^{\prime}=\operatorname{Conv}\left\{\left(\left[\begin{array}{cccc}
x_{k}^{1} & x_{k}^{2} & \cdots & x_{k}^{n} \\
\vdots & \vdots & \ddots & \vdots \\
x_{2}^{1} & x_{2}^{2} & \cdots & x_{2}^{n} \\
x_{1}^{1} & x_{1}^{2} & \cdots & x_{1}^{n}
\end{array}\right], g\right) \in M_{k, n} \times \mathbb{N} \backslash\{0\} \mid \begin{array}{l}
\sum_{l=1}^{k} x_{l}^{i}=1, \forall i \in \llbracket 1, n \rrbracket, \\
g \geq \max _{i \in \llbracket 1, n \rrbracket} \sum_{l=1}^{k} l x_{l}^{i},
\end{array}\right\}
$$

A full description of $Q$ is given below.

\section{Theorem 7}

$$
Q=\left\{\begin{array}{l}
\sum_{l=1}^{k} y_{l}^{i}=1, \forall i \in \llbracket 1, n \rrbracket, \\
\sum_{l=2}^{k} \sum_{i=1}^{n} \lambda_{l}^{i} y_{l}^{i} \geq h-1, \forall \lambda \in \Lambda, \\
y_{l}^{i} \geq 0, \forall(i, l) \in \llbracket 1, n \rrbracket \times \llbracket 1, k \rrbracket, h \geq 1 .
\end{array}\right.
$$

Proof. It is a simple fact that $h \geq 1$ is the only possible facet of type (3) while the positivity constraints are the only possible facets of type (1). Let us consider an inequality of type (2) defining a facet of $Q$. Any extreme point of $Q$ saturating such a facet necessarily satisfies $h=\min _{i \in \llbracket 1, n \rrbracket} \sum_{l=1}^{k} l y_{l}^{i}$ implying that it is also a point of $P$. Using this observation and the fact that $Q$ and $P$ have the same dimension, we deduce that any facet of $Q$ of type (2) is also a facet of $P$. Using the description of $P$, we get the result.

Similarly to Theorem 6 we can deduce that the separation problem with respect to $Q$ is solvable in polynomial time as well.

We can also derive a full description of $P^{\prime}$ and $Q^{\prime}$ from the previous results.

\section{Theorem 8}

$$
\begin{aligned}
P^{\prime}=\left\{\begin{array}{l}
\sum_{l=1}^{k} x_{l}^{i}=1, \forall i \in \llbracket 1, n \rrbracket, \\
\sum_{l=1}^{k-1} \sum_{i=1}^{n} \lambda_{l}^{i} x_{l}^{i} \leq g-k, \forall \lambda \in \tilde{\Lambda}, \\
\sum_{l=g_{\min }+1}^{k} \sum_{i=1}^{n}\left(l-g_{\min }\right) x_{l}^{i}+g_{\min } \geq g, \forall g_{\min } \in \llbracket 1, k \rrbracket, \\
x_{l}^{i} \geq 0, \forall(i, l) \in \llbracket 1, n \rrbracket \times \llbracket 1, k \rrbracket, g \in \mathbb{R},
\end{array}\right. \\
Q^{\prime}=\left\{\begin{array}{l}
\sum_{l=1}^{k} x_{l}^{i}=1, \forall i \in \llbracket 1, n \rrbracket, \\
\sum_{l=1}^{k-1} \sum_{i=1}^{n} \lambda_{l}^{i} x_{l}^{i} \leq g-k, \forall \lambda \in \tilde{\Lambda}, \\
x_{l}^{i} \geq 0, \forall(i, l) \in \llbracket 1, n \rrbracket \times \llbracket 1, k \rrbracket, g \leq k
\end{array}\right.
\end{aligned}
$$

where

$$
\tilde{\Lambda}=\left\{\begin{array}{l|l}
\lambda=\left(\lambda_{l}^{i}\right)_{(i, l) \in \llbracket 1, n \rrbracket \times \llbracket 1, k \rrbracket} \in \mathbb{N}^{n k} & \begin{array}{l}
\lambda_{l+1}^{i} \leq \lambda_{l}^{i}, \forall(i, l) \in \llbracket 1, n \rrbracket \times \llbracket 1, k-1 \rrbracket \\
\sum_{i=1}^{n} \lambda_{l}^{i}=k-l, \forall l \in \llbracket 1, k \rrbracket
\end{array}
\end{array}\right\} .
$$

Proof. Take an extreme point $(y, h)$ of $P$, and let $(x, g) \in M_{k, n} \times \mathbb{N} \backslash\{0\}$ such that $x_{l}^{i}=$ $y_{k-l+1}^{i}, \forall(i, l) \in \llbracket 1, n \rrbracket \times \llbracket 1, k \rrbracket$ and $g=k-h+1$, then $g=\max _{i \in \llbracket 1, n \rrbracket} \sum_{l=1}^{k} l x_{l}^{i}$, hence $(x, g) \in P^{\prime}$. Conversely, any extreme point $(x, g)$ of $P^{\prime}$ can be obtained from an extreme point of $P$ in this manner. So $P^{\prime}$ is obtained from $P$ doing the change of variables $x_{l}^{i}=$ $y_{k-l+1}^{i}, \forall(i, l) \in \llbracket 1, n \rrbracket \times \llbracket 1, k \rrbracket$ and $g=k-h+1$. Therefore its hyperplane representation is obtained from that of $P$ in Theorem 5. Similarly, $Q^{\prime}$ is obtained from $Q$ doing the same change of variables and its hyperplane representation is thus obtained from Theorem 7. 
The previous results imply that the separation problems related to $P^{\prime}$ and $Q^{\prime}$ can be solved in polynomial time.

\section{Conclusion}

In this paper we exhibited a family of polyhedra emerging in very diverse combinatorial optimization problems including the most imbalanced orientation of a graph, the minimum span frequency assignment and some scheduling problems. Then a full description of these polyhedra has been derived. We also proved that the separation problems related to these polyhedra can be solved in polynomial time and thus optimization over them can be done in polynomial time.

We think that many combinatorial optimization problems where discretization techniques are used can benefit from the description of the polyhedra introduced in this paper. We are currently carrying out experimentations to study the efficiency of cutting plane algorithms based on these polyhedra. Future work may be directed towards investigations on extensions of the polyhedra we considered here in order to get better approximations while still keeping the feature of computational tractability. One can, for example, study $\{0,1\}$ assignment matrices appended with both $h$ and $g$ (the index of the lowest (resp. highest) nonzero row of the matrix). The related polytope is included in the intersection of $P$ or $Q$. Some preliminary investigations show that more inequalities are necessary to describe the polytope.

\section{References}

1. W. Ben-Ameur, A. Glorieux \& J. Neto: 'On the most imbalanced orientation of a graph', Proceedings of COCOON2015, LNCS (2015)

2. W. Cook, W. Cunningham, W. Pulleyblank \& A. Schrijver: 'Combinatorial Optimization', ISBN: 978-0-471-55894-1 (1997)

3. M. Garey \& D. Johnson: 'Computers and Intractability; A Guide to the Theory of NPCompleteness', W.H.Freeman \& Co. (1990)

4. D. Hochbaum \& D. Shmoys: 'A polynomial approximation scheme for scheduling on uniform processors: Using the dual approximation approach' SIAM Journal on Computing 17(3), 539-551 (1988)

5. E. Horowitz \& S. Sahni: 'Exact and approximate algorithms for scheduling nonidentical processors', Journal of ACM 23(2), 317-327 (1976)

6. R. Karp: 'Reducibility Among Combinatorial Problems', Complexity of Computer Computations, Plenum Press (1972)

7. A. Koster: 'Frequency Assignment - Models and Algorihtms' PhD thesis, Univ. Maastricht, the Netherlands (1999)

8. Z. Mann \& A. Szajkó: 'Complexity of different ILP models of the frequency assignment problem' Linear Programming - New frontiers in Theory and Applications, 305-326 (2012)

9. P. Martins: 'Extended and discretized formulations for the maximum clique problem' Computers \& Operations Research 37(7), 1348-1358 (2011)

10. V. Vazirani: 'Minimum Makespan Scheduling' Approximations Algorithms, Springer, Chapter 10, 79-83 (2003)

11. L. Wolsey: 'Integer Programming', ISBN: 978-0-471-28366-9 (1998) 